\title{
Contrast Spread in the Superoposterior Approach of Transforaminal Epidural Steroid Injections for Lumbosacral Radiculopathy
}

Young Cheol Jeong, MD, Chung Ho Lee, MD, Seok Kang, MD, Joon Shik Yoon, MD, PhD

Department of Physical Medicine and Rehabilitation, Korea University Guro Hospital, Seoul, Korea

\begin{abstract}
Objective To observe the contrast spread in superoposterior transforaminal epidural steroid injection (SP TFESI) and investigate the correlation between spread patterns and efficacy.

Methods Thirty-one patients with lumbosacral radiculopathy underwent single-level TFESI under fluoroscopy. The final needle tip position was targeted toward the SP quadrant of the intervertebral foramen. To observe the spread, $1 \mathrm{~mL}$ of contrast material was injected, followed by a steroid injection. The contrast spread was graded anteroposteriorly and vertically in the epidural space. The effect of SP TFESI was evaluated by proportional pain score reduction.

Results Levels injected were L4-5 ( $\mathrm{n}=20)$ and L5-S1 $(\mathrm{n}=11)$. Seventeen cases were lateral, and 14 were central herniated disc (HD). Baseline mean visual analog scale score was 6.23. Contrast dispersed dorsally in all the cases, and $45.2 \%$ cases showed a concurrent ventral spread. The proportion of the pain reduction after 2 weeks showed no difference between the two groups. In vertical spreading analysis, mean cephalic/caudal grades were 1.40/1.55 at L4-5 level and 1.73/1.64 at L5-S1 level. The HD location had no effect on contrast dispersion.

Conclusion In SP TFESI, ventral contrast spread did not guarantee a better effect; however, the extent of cephalic flow in ventral expansion group correlated with the proportion of pain reduction.
\end{abstract}

Keywords Epidural injection, Contrast media, Lumbosacral, Radiculopathy, Treatment outcome

\section{INTRODUCTION}

Epidural steroid injection (ESI) is a widely used interventional treatment for low back pain caused by lumbosacral radiculopathy $[1,2]$. Among the ESI techniques, the trans- foraminal approach is considered as a preferred method for effective delivery of the injectate to the ventral epidural space, close to the dorsal root ganglion [1-4]. Conventionally, when transforaminal epidural steroid injections (TFESI) are administered, the needle is placed below

Received June 13, 2016; Accepted October 3, 2016

Corresponding author: Joon Shik Yoon

Department of Physical Medicine and Rehabilitation, Korea University Guro Hospital, 148 Gurodong-ro, Guro-gu, Seoul 08308, Korea. Tel: +82-2-26261500, Fax: +82-2-2626-1513, E-mail: rehab46@korea.ac.kr

ORCID: Young Cheol Jeong (http://orcid.org/0000-0003-2619-3640); Chung Ho Lee (http://orcid.org/0000-0001-5140-2841); Seok Kang (http://orcid. org/0000-0001-5614-4175); Joon Shik Yoon (http://orcid.org/0000-0002-8985-279X).

(c) This is an open-access article distributed under the terms of the Creative Commons Attribution Non-Commercial License (http://creativecommons.org/ licenses/by-nc/4.0) which permits unrestricted noncommercial use, distribution, and reproduction in any medium, provided the original work is properly cited. Copyright (c) 2017 by Korean Academy of Rehabilitation Medicine 
the underside of the pedicle and above the exiting spinal nerve root, within the upper half of the neural foramen, namely called as 'safe triangle.' Specifically, the target of the needle is the superoanterior (SA) quadrant of the neural foramen. However, this conventional technique occasionally encounters the following problems. (1) Because the needle must traverse the nerve root to reach the target, chances of touching the nerve root is highly probable. (2) In a severely degenerated and narrowed foramen, accessing the epidural space is very hard [5]. Furthermore, since the radicular artery (the artery of Adamkiewicz) are most commonly in the SA quadrant of the foramen [6-9], these arteries can be punctured, causing catastrophic complications [10,11]. Therefore, an alternative technique has been proposed in which the target of the needle is superoposterior (SP) quadrant of the foramen [5,12-14]. A previous study reported that the ventral flow and extent of contrast medium spreading resulting in SA TFESI [15]. However, in SP TFESI, because the needle is placed at the posterior aspect of the foramen, it is unclear whether the medication can reach the ventral epidural space and relieve pain [5].

This study aimed to observe the contrast spread pattern in SP TFESI and investigate the correlation between the spread pattern and clinical efficacy. In addition, the relationship between the anatomical pathology and contrast spread patterns in SP TFESI was analyzed.

\section{MATERIALS AND METHODS}

\section{Subjects}

This study was conducted from November 2014 to December 2015. We recruited 31 subjects diagnosed with lumbar radiculopathy caused by a herniated intervertebral disc, undergoing TFESI. Each patient complained of persistent low back pain and radicular pain in the lower extremity for at least 2 weeks despite appropriate medication and conservative treatment. Diagnosis was confirmed by physical examination, electrophysiological study, and magnetic resonance (MR) images. Patients $<18$ years, $>80$ years, or pregnant were excluded from the study. Other exclusion criteria were as follows: (1) history of previous lumbar surgery, (2) presence of systemic inflammatory disease, (3) uncontrolled diabetes, (4) allergy to contrast medium or lidocaine, and (5) injections within 3 months. Patients were allowed to take medica- tions prescribed before the study; however, additional pain medications were not prescribed during the study.

This study was approved by the regional Institutional Review Board, and written informed consents were obtained from all the patients.

\section{MR image analysis}

The primary pathology causing radiculopathy was determined according to the report of the radiologists of the author's hospital and classified as central herniated disc (HD) or lateral HD for further analysis of the structures. In accordance with the 'zone system' [16], central HD was defined as the HD medial to the medial edge of the articular facets, and lateral HD was defined as the HD lateral to the medial edge of the articular facets.

\section{Procedure}

All epidural injections were administered in an outpatient procedure room by one of two physicians with extensive experience in fluoroscopically guided lumbar TFESI. The criteria for choosing the candidate target levels were determined by the HD location of the MR images and clinical symptoms as follows: (1) Patients with lateral HD at L4-5 level on MR images underwent L4-5 TFESI. (2-1) Patients with central HD at L4-5 level and symptoms of L5 radiculopathy underwent L5-S1 TFESI. (2-2) In case of difficulty in approaching the L5-S1 foramen despite central HD at L4-5 level, TFESI was performed using the $L 4-5$ foramen instead. Some studies $[17,18]$ and our own clinical experience reported a successful therapeutic effect with TFESI performed by substituting the foramen one level above. (3) The patients with lateral and central HD at L5-S1 level and symptom of L5 radiculopathy underwent L5-S1 TFESI.

Subjects were placed in the prone position on a fluoroscopy table, and the skin was prepared in a sterile manner with an iodine antiseptic solution and draped. The Carm was set to optimally visualize the target foramen, rotated in the range $20^{\circ}-25^{\circ}$ to the lesion side to provide an oblique view, and tilted cranially or caudally at $0^{\circ}-5^{\circ}$ if needed to align the vertebral end plates. The overlying soft tissue was anesthetized using $2 \%$ lidocaine $\mathrm{HCl}$ (Huons, Hwaseong, Korea). A 23-gauge, 3.5-inch spinal needle with a curved tip was advanced into the region of the involved nerve root under fluoroscopic guidance. As this study aimed to evaluate only SP TFESI, the final 
needle tip position was targeted towards the SP quadrant of the intervertebral foramen, where the needle was placed under the median inferior edge of the pedicle on oblique view, and anterior to the posterior margin of the intervertebral foramen on lateral view. To confirm the exact injection position and observe the pattern of spread, $1 \mathrm{~mL}$ of contrast material Xenetix 300 (Guerbet, Gorinchem, The Netherlands) was injected. Furman et al. [19] demonstrated that when $1 \mathrm{~mL}$ of contrast (ISOVUE 320; Bracco Diagnostics, Princeton, NJ, USA) was used on SA TFESI, the contrast reached the superior aspect of the superior intervertebral disc (IVD) or inferior aspect of the inferior IVD in $46 \%$ of the total attempts, for each landmark. Thus, we assumed that $1 \mathrm{~mL}$ of contrast would also be sufficient to observe the contrast spread in SP TFESI. After the needle was confirmed in the SP quadrant position without intravascular flow or soft tissue infiltration and the contrast spread was assessed, steroidal drug was injected. The drug composed of $2 \mathrm{~mL}$ of $0.5 \%$ lidocaine, $0.5 \%$ lidocaine $\mathrm{HCl}$ (Samjin, Hwaseong, Korea), and 20 $\mathrm{mg}(0.5 \mathrm{~mL})$ of triamcinolone acetonide (Triam; Shinpoong, Seoul, Korea) and was slowly administered.

\section{Measurements}

General characteristics such as age, sex, height, weight, and body mass index of all the patients were evaluated. Prior to the TFESI, the baseline pain score was evaluated

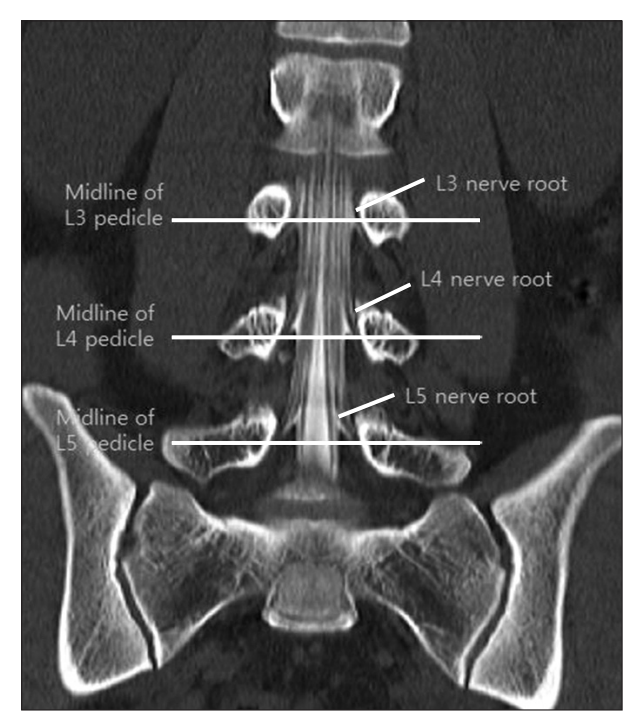

Fig. 1. CT myelography showing L3, L4, and L5 nerve roots. Openings of the dural sleeves span the upper half of the pedicles at L3, L4, and L5 vertebrae. CT, computed tomography. using the visual analog scale (VAS). At 2 weeks after TFESI, the follow-up VAS score was measured. To compare the clinical efficacy according to the spreading pattern, the percentage of the pain relief was calculated using the following formula: (baseline pain score - follow-up pain score $) \times 100 /$ baseline pain score.

Ventral and dorsal contrast spread patterns were analyzed according to the following criteria. Ventral spreading, contrast medium reached the posterior aspect of the vertebral body or intervertebral disc; dorsal spreading, contrast medium reached the lamina or ligamentum flavum. In addition, vertical spread patterns for the cephalic and caudal directions were separately evaluated. A grading system was used for the extent of contrast spread to a predetermined position by adopting Furman's concept [19], emphasizing the importance of various relevant pathologic landmarks in lumbar radiculopathy. The IVD is important to a central HD. As the opening of the dural sleeve spans the upper half of the pedicles at L3, L4, and L5 vertebrae, the vertical midpoint of the pedicle was also considered to be an important landmark for the entrance between the central canal and spinal root (Fig. 1). The grading system was as follows (Fig. 2): Grade 0 , no visible spread; Grade 1, within the same vertebral level; Grade

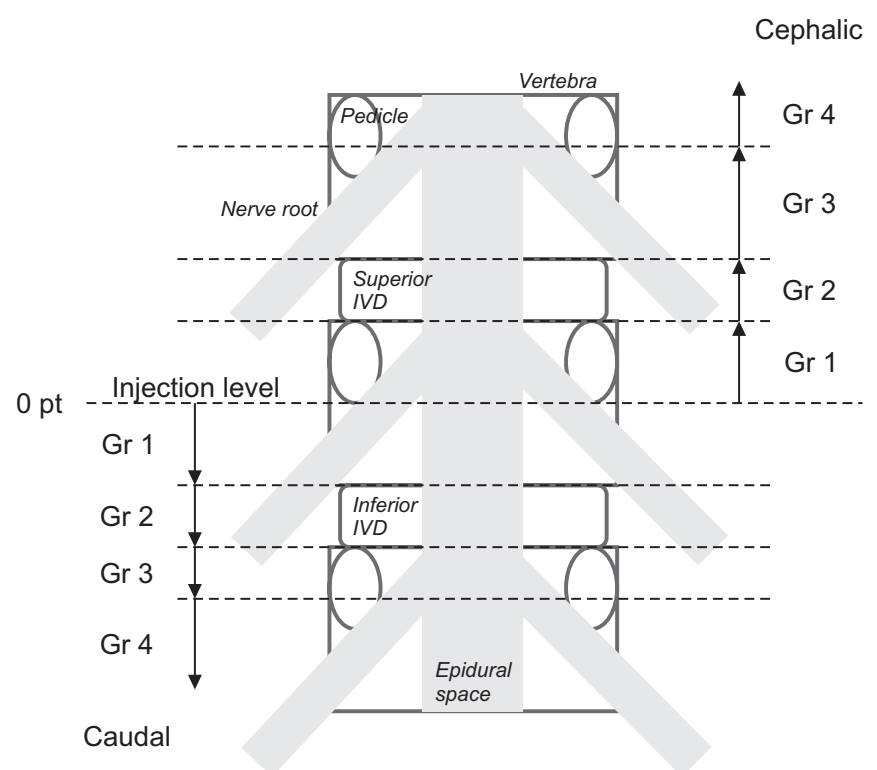

Fig. 2. Vertical spread grading system on a schematic drawing of lumbar vertebra with epidural spaces (anteroposterior view). Grade was determined by the spreading extent of contrast from injection level in both the cephalic and caudal directions separately. IVD, intervertebral disc. 
2, within the vertical level of the superior IVD (cephalad) or inferior IVD (caudad); Grade 3, above the superior IVD, not exceeding the horizontal midline of the upper level pedicle, or below the inferior IVD, not exceeding the horizontal midline of the lower level pedicle; Grade 4, over the horizontal midline of the upper or lower pedicle. The grade of spreads was separately evaluated in cephalic and caudal directions (Fig. 3). In the case of dorsal isolated spread, the grade was derived from the dorsal spread, and in the case of concurrent ventral and dorsal spread (ventral expansion), the grade was derived from the highest one between the ventral and dorsal spreads.

\section{Statistical analysis}

The distribution of anteroposterior patterns of contrast spread was examined according to the isolated ventral, dorsal, or concurrent spread. Using the odds ratio (OR), the differential likelihood of anteroposterior contrast spread at each injection level was determined. Student t-test was used to compare the treatment effect in each group showing different contrast spread according to the ventral and dorsal sides of the epidural space. Pearson correlation analysis was performed to reveal the association between the treatment effect and extent of vertical contrast spread. Fisher exact test was used to investigate the correlation between the lesion location (lateral or central HD) and contrast spread. Statistical analyses were performed using the SPSS software package ver. 12.0 (SPSS Inc., Chicago, IL, USA). The p-values $<0.05$ were considered to indicate statistical significance.

\section{RESULTS}

General characteristics of all the patients are listed in Table 1. TFESI was performed at the L4-5 and L5-S1 levels in 20 and 11 patients, respectively. The main pathology at the injected level was lateral HD in 17 patients and central HD in 14. Fifteen patients had a lateral HD at the L4-5 level on MR images and underwent L4-5 TFESI. Of these, only one patient was confirmed to have L4 radiculopathy by an electrophysiologic study, and five had L5 radiculopathy. The others had normal electrophysiologic findings, but complained of symptoms of L5 radiculopathy. Five patients with central HD at L4-5 level were suspected to have L5 radiculopathy, but underwent L4-5 TFESI because of anatomical changes in the lumbosacral area: 3 cases with a high iliac crest, 1 case of a large L5 transverse process, and 1 case of pseudo-arthrosis of L5 vertebra with the iliac crest. Six patients with central HD at L4-5 level and 2 with lateral HD at L5-S1 level underwent L5-S1 TFESI. Three patients had central HD at L5-S1 level, but presented symptoms suggesting L5 radiculopathy and underwent L5-S1 TFESI (Supplement Table S1 shows clinical presentations, the results of MRI and EMG,
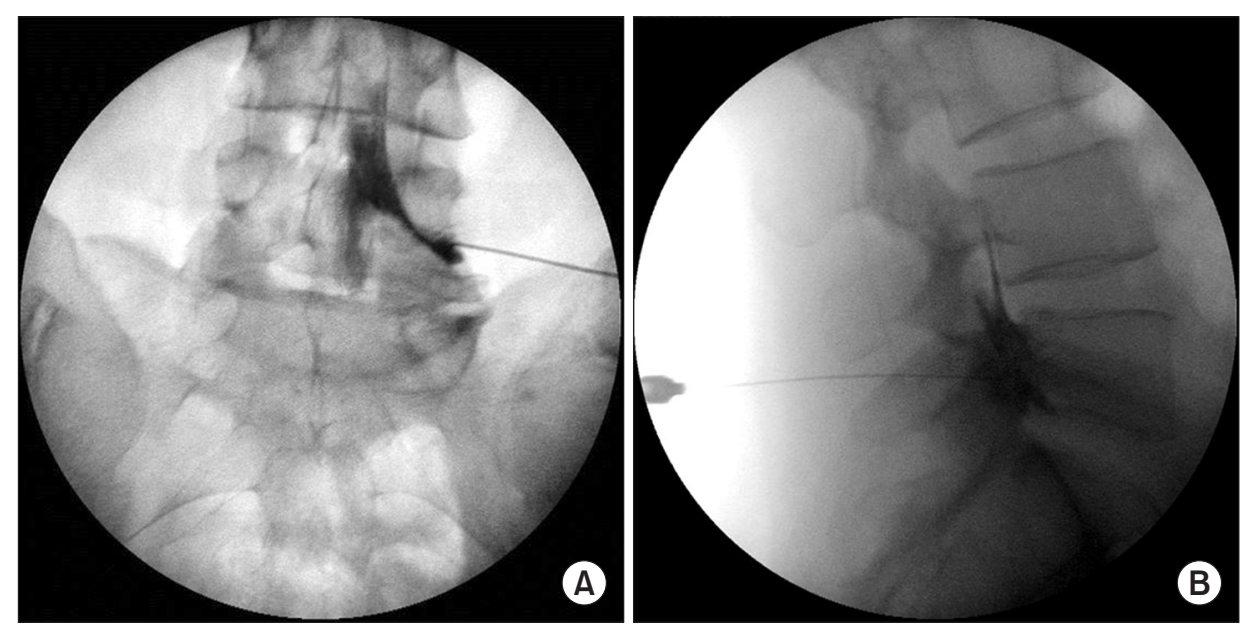

Fig. 3. Anteroposterior (A) and lateral (B) fluoroscopic view of L5-S1 SP TFESI. Contrast filled the dorsal epidural space and spread to the ventral space. Vertical dispersal analysis showed Grade 3 (above the superior IVD, not exceeding the horizontal midline of the upper level pedicle) in the cephalic direction and Grade 2 (within the vertical level of the inferior IVD) in the caudal direction. SP TFESI, superoposterior transforaminal epidural steroid injection; IVD, intervertebral disc. 
and ESI-performed level for each patient). The mean VAS score of all the patients was $6.23 \pm 1.33$ at baseline.

The contrast medium spread dorsally in all cases $(\mathrm{n}=31)$, and $45.2 \%$ (14 of 31) showed concurrent ventral spread. However, there was no isolated contrast filling of the ventral epidural space. In addition, when contrast patterns in the L4-5 and L5-S1 levels were separately analyzed, a

Table 1. Demographics of the subjects $(\mathrm{n}=31)$

\begin{tabular}{|cc|}
\hline Parameter & Value \\
\hline Sex & 12 \\
\hline Male & 19 \\
\hline Female & $63.3 \pm 14.6(30-85)$ \\
\hline Age (yr) & 20 \\
\hline Injection level & 11 \\
\hline L4-5 & \\
\hline L5-S1 & 13 \\
\hline Injection side & 18 \\
\hline Right & 17 \\
\hline Left & 14 \\
\hline Lesion type & \\
\hline Lateral HD & $6.23 \pm 1.33(4-9)$ \\
\hline Central HD & \\
\hline VAS at baseline & \\
\hline
\end{tabular}

Values are presented as number or mean \pm standard deviation (range).

HD, herniated disc; VAS, visual analog scale. significantly greater tendency was observed for the contrast to expand ventrally at the L5-S1 (8 of 11) level than at the L4-5 (6 of 20) level, according to the results of Fisher exact test $(\mathrm{p}=0.031, \mathrm{OR}=6.2)$.

In the analysis of efficacy of SP TFESI, whether or not ventral expansion of contrast was present, the changes in the VAS scores and the proportion of pain reduction at 2 weeks after SP TFESI were not statistically different ( $\mathrm{p}>0.1)$ (Table 2).

In the vertical contrast spread analysis, at L4-5 level, the mean grade of contrast spread was $1.40 \pm 1.05$ in a cephalic direction and $1.55 \pm 1.32$ in a caudal direction. At L5-S1 level, the mean grade was $1.73 \pm 1.19$ and $1.64 \pm 0.92$ in the cephalic and caudal directions, respectively. In an analysis of the correlation between the vertical spread pattern and treatment effect according to the dorsoventral spread (dorsal isolated and ventral expansion), the grades for cephalic extension in the ventral expansion group showed a meaningful positive correlation with proportional pain reduction $(\mathrm{r}=0.676, \mathrm{p}<0.01)$ (Table 3 ).

The changes in the VAS scores along with the degree of vertical contrast spread on each direction is summarized, and listed in Table 4.

In addition, the location of HD (central or lateral HD) was not found to affect the anteroposterior spread of contrast in the epidural space at any level (Fisher exact test, both $\mathrm{p}>0.1$ for L4-5 and L5-S1).

In the extent of vertical spread, the mean grades for

Table 2. VAS of ventral expansion group and dorsal isolated group treated with SP TFESI

\begin{tabular}{|c|c|c|c|c|c|}
\hline \multirow{3}{*}{ Injection level } & \multirow{3}{*}{ Contrast pattern } & \multicolumn{4}{|c|}{ VAS } \\
\hline & & \multirow{2}{*}{ Pre } & \multirow{2}{*}{ After 2 weeks } & \multicolumn{2}{|c|}{ Reduction } \\
\hline & & & & $\Delta$ & Rate (\%) \\
\hline \multirow[t]{3}{*}{ L4-5 } & Ventral expansion $(n=6)$ & $6.3 \pm 1.5$ & $3.0 \pm 0.9$ & -3.33 & 52.8 \\
\hline & Dorsal isolated $(\mathrm{n}=14)$ & $5.9 \pm 1.4$ & $2.9 \pm 1.0$ & -2.93 & 49.9 \\
\hline & p-value & 0.505 & 0.882 & 0.394 & 0.619 \\
\hline \multirow[t]{3}{*}{ L5-S1 } & Ventral expansion $(\mathrm{n}=8)$ & $6.6 \pm 1.3$ & $3.6 \pm 0.9$ & -3.00 & 45.4 \\
\hline & Dorsal isolated $(\mathrm{n}=3)$ & $6.7 \pm 0.6$ & $4.0 \pm 1.7$ & -2.67 & 40.3 \\
\hline & p-value & 0.960 & 0.641 & 0.628 & 0.596 \\
\hline \multirow[t]{3}{*}{ Total } & Ventral expansion $(n=14)$ & $6.5 \pm 1.3$ & $3.4 \pm 0.9$ & -3.14 & 48.6 \\
\hline & Dorsal isolated $(\mathrm{n}=17)$ & $6.0 \pm 1.3$ & $3.1 \pm 1.2$ & -2.88 & 48.2 \\
\hline & p-value & 0.307 & 0.539 & 0.434 & 0.941 \\
\hline
\end{tabular}

Values are presented as mean \pm standard deviation.

Student t-test was used to compare the values for each contrast pattern group.

SP TFESI, superoposterior transforaminal epidural steroid injection; VAS, visual analog scale; $\Delta$, changes in VAS value from before and 2 weeks after TFESI. 
cephalic and caudal spreads showed no statistical difference between the central and lateral HD, when analyzed according to each level and direction of spread (Student t-test).

No major complications were reported.

\section{DISCUSSION}

Recently, several studies reported that SP TFESI is a safer alternative to the conventional SA approach, because of the lower risk of vascular complications [20]. This study aimed to investigate the contrast spreading pattern and its correlation with efficacy for SP TFESI. Contrast was examined for the spread to both the ventral and dorsal epidural spaces, and in cephalic and caudal directions. Clinical efficacy was significantly correlated with the cephalic spreading pattern. These results indicate that the spreading of injectate could be one of the factors determining the clinical efficacy in SP TFESI.

Several previous studies reported conflicting results for differences in clinical efficacy between SA and SP TFESI.

Table 3. Pearson correlation analysis for vertical dispersal extent and degree of proportional pain reduction with SP TFESI according to dorsoventral contrast pattern type

\begin{tabular}{ccc}
\hline Contrast pattern type & Cephalic & Caudal \\
\hline Dorsal isolated $(\mathrm{n}=17)$ & 0.434 & 0.187 \\
p-value & 0.082 & 0.473 \\
Ventral expansion $(\mathrm{n}=14)$ & $0.676^{*}$ & -0.370 \\
p-value & 0.008 & 0.192 \\
\hline
\end{tabular}

SP TFESI, superoposterior transforaminal epidural steroid injection.

${ }^{*} \mathrm{p}<0.01$.
$[5,15]$. Desai et al. [15] reported that the efficacy of SA TFESI was superior to that of SP TFESI and suggested that ventral and greater extent of contrast spread would assure better therapeutic efficacy. However, in this study, the ventral spreading pattern was not correlated with the clinical efficacy in SP TFESI. The ventral spreading of contrast was observed in $45.2 \%$ of the cases in this study, which is less than the $61.4 \%$ in the study by Desai et al. [15]. In addition, ventral expansion was more commonly observed at the L5-S1 level than at the L4-5 level. According to a study on the morphometrics of the lumbar spinal canal, the lower lumbar spinal canal has a greater midsagittal and interpedicular diameter through L3 to L5 level [21]. The spinal canal space at the L5-S1 level is larger than the space at the L4-5 level, and the less constricting epidural space may permit easier ventral expansion of contrast. Since TFESI was performed more frequently at the L4-5 level than at the L5-S1 level, we assumed that ventral spreading patterns were observed in a relatively small number of patients in this study. However, further research is needed to determine the correlation between the differences in the epidural space and contrast spreading patterns.

Several previous studies examined the vertical direction of contrast spread in TFESI. Two studies reported a tendency for more cephalic spread [15,22]. However, Vassiliev [17] and Gajraj [23] reported preferential spread of contrast onto the medially located nerve roots (lower level of nerve root). Furman et al. [19] observed an equivalent extent of spread in both the directions in the presence of $1 \mathrm{~mL}$ of contrast. In this study, the contrast medium spread to a similar degree on our grading system in both the cephalic and caudal directions.

The vertical contrast spread pattern analysis showed

Table 4. Mean of VAS changes according to the degree of vertical extent of contrast spreads

\begin{tabular}{|c|c|c|c|c|c|c|}
\hline \multirow{3}{*}{$\begin{array}{c}\text { Degree of vertical } \\
\text { spread }\end{array}$} & \multicolumn{6}{|c|}{ Mean of VAS changes } \\
\hline & \multicolumn{2}{|c|}{ L4-5 } & \multicolumn{2}{|c|}{ L5-S1 } & \multicolumn{2}{|c|}{ Total } \\
\hline & Cephalic & Caudal & Cephalic & Caudal & Cephalic & Caudal \\
\hline Grade 0 & $-2.50(4)$ & $-2.80(5)$ & (0) & $-4.00(1)$ & $-2.50(4)$ & $-3.00(6)$ \\
\hline Grade 1 & $-2.57(7)$ & $-3.40(5)$ & $-2.71(7)$ & $-2.50(4)$ & $-2.64(14)$ & $-3.00(9)$ \\
\hline Grade 2 & $-3.57(7)$ & $-2.71(7)$ & $-3.00(2)$ & $-3.25(4)$ & $-3.44(9)$ & $-2.91(11)$ \\
\hline Grade 3 & $-4.00(1)$ & (0) & (0) & $-2.50(2)$ & $-4.00(1)$ & $-2.50(2)$ \\
\hline Grade 4 & $-4.00(1)$ & $-3.67(3)$ & $-3.50(2)$ & (0) & $-3.67(3)$ & $-3.67(3)$ \\
\hline
\end{tabular}

The numbers of patients with each degree of vertical spread are in parentheses. VAS, visual analog scale. 
a significant correlation between the cephalad spreading and clinical efficacy in the ventral expansion group. As mentioned above, Desai et al. [15] confirmed that the more level of vertebra covered by the contrast would lead to a better clinical effect. More amount of medication passed into the epidural space would eventually lead to increased flow of medication into the adjacent nerve roots as well as the nerve root of the target level. In concordance to this, we assume that the positive correlation between the vertical contrast extent and therapeutic effect is a reasonable phenomenon.

In this study, since most patients who underwent L4-5 TFESI were thought to have L5 radiculopathy, the therapeutic effects might not be the direct results of TFESI on the L4 nerve root. In these cases, more medial spread of medication might lead to a more successful reduction of the L5 root symptoms, because the L5 root is located medial to the L4 root at L4-5 level. According to the study of Vassiliev [17], volume-spread relationships were observed, when L4-5 TFESI was performed. Contrast spread onto the L5 nerve root in $46.1 \%$ with $1 \mathrm{~mL}$ of contrast and in $61.5 \%$ with $2-3 \mathrm{~mL}$ of contrast. No spread of contrast was observed onto the L3 nerve root. In this study, though we used $1 \mathrm{~mL}$ of contrast for evaluating the contrast spread patterns, $2.5 \mathrm{~mL}$ of medication was used for the therapeutic purpose, and such volume would sufficiently affect the L5 nerve root with L4-5 TFESI.

The effect of positional type of HD (central or lateral) was also evaluated on the contrast spread. Prior studies showed the similar results. Botwin et al. [22] reported no significant difference in the overall flow patterns between the patients with lumbar spinal stenosis and HD by using conventional TFESI. Furman et al. [19] found no statistical significance in the contrast volume needed to reach specific landmarks with or without the presence of central or foraminal stenosis. Similar to prior studies, SP TFESI with central and lateral HD showed did not show any statistical differences in the contrast spread in the ventral or dorsal direction or in vertical extent of spread.

This study has the following limitations. First, contrast has a higher viscosity than the medication. This difference can cause unexpected spread patterns in the injected medication. Moreover, we only captured a fluoroscopic image immediately after the completion of SP TFESI. Because both the contrast and medication would spread further over time, we may have under-estimated the ex- tent of dispersion. However, because the lower viscosity of the medication and the time effect would result in at least equivalent or larger dispersion, we think that our interpretation is still valid or can be extended. If extended, based on adequate spreading, the significance of dividing the anterior and posterior epidural space for evaluation of contrast dispersion would lessen and enhance our result, thereby showing no difference in the proportion of pain reduction between the dorsal spreading and ventral expansion. Another limitation is that the pathologic level of radiculopathy was not clear in some patients. No definite correspondence was observed between the electrophysiologic study, MR images, and clinical symptoms. If cases with more definitive levels of radiculopathy are recruited in a larger study, the results would be demonstrated more clearly.

In SP TFESI, achieving ventral contrast spread did not guarantee better treatment effects; however, that the extent of cephalic spread in the ventral expansion group was positively correlated with the proportion of pain reduction.

Since this study analyzed contrast spread patterns, it should provide data for use in further research. In particular, because SP TFESI is mainly used in difficult cases with severely degenerated vertebrae, it would be meaningful to compare the therapeutic efficacy of SP TFESI and SA TFESI in the patients with severe foraminal stenosis in future study.

\section{SUPPLEMENTARY MATERIALS}

Supplementary materials can be found via http://doi. org/10.5535/arm.2017.41.3.413. Table S1. Patients' clinical presentations, results of MRI and EMG, and epidural steroid injection level.

\section{CONFLICT OF INTEREST}

No potential conflict of interest relevant to this article was reported.

\section{REFERENCES}

1. Manchikanti L, Staats PS, Singh V, Schultz DM, Vilims $\mathrm{BD}$, Jasper JF, et al. Evidence-based practice guidelines for interventional techniques in the manage- 
ment of chronic spinal pain. Pain Physician 2003;6:381.

2. Boswell MV, Hansen HC, Trescot AM, Hirsch JA. Epidural steroids in the management of chronic spinal pain and radiculopathy. Pain Physician 2003;6:319-34.

3. Ackerman WE 3rd, Ahmad M. The efficacy of lumbar epidural steroid injections in patients with lumbar disc herniations. Anesth Analg 2007;104:1217-22.

4. Manchikanti L. Transforaminal lumbar epidural steroid injections. Pain Physician 2000;3:374-98.

5. Lee IS, Kim SH, Lee JW, Hong SH, Choi JY, Kang HS, et al. Comparison of the temporary diagnostic relief of transforaminal epidural steroid injection approaches: conventional versus posterolateral technique. AJNR Am J Neuroradiol 2007;28:204-8.

6. Devereaux MW. Anatomy and examination of the spine. Neurol Clin 2007;25:331-51.

7. Adamkiewicz A. Die blutgefasse des menschlichen ruckenmarkes. II Theil: Die gefasse der ruckenmarksoberflache. S B Heidelberg Akad Wiss 1882;85:10130.

8. Kirkaldy-Willis WH, Bernard TN. Managing low back pain. 4th ed. London: Churchill Livingstone; 1999.

9. Murthy NS, Maus TP, Behrns CL. Intraforaminal location of the great anterior radiculomedullary artery (artery of Adamkiewicz): a retrospective review. Pain Med 2010;11:1756-64.

10. Lyders EM, Morris PP. A case of spinal cord infarction following lumbar transforaminal epidural steroid injection: MR imaging and angiographic findings. AJNR Am J Neuroradiol 2009;30:1691-3.

11. Somayaji HS, Saifuddin A, Casey AT, Briggs TW. Spinal cord infarction following therapeutic computed tomography-guided left L2 nerve root injection. Spine (Phila Pa 1976) 2005;30:E106-8.

12. Jasper JF. Lumbar retrodiscal transforaminal injection. Pain Physician 2007;10:501-10.

13. Glaser SE, Shah RV. Root cause analysis of paraplegia following transforaminal epidural steroid injections: the 'unsafe' triangle. Pain Physician 2010;13:237-44.
14. Helm S, Glaser S, Falco F, Henry B. A medical-legal review regarding the standard of care for epidural injections, with particular reference to a closed case. Pain Physician 2010;13:145-50.

15. Desai MJ, Shah B, Sayal PK. Epidural contrast flow patterns of transforaminal epidural steroid injections stratified by commonly used final needle-tip position. Pain Med 2011;12:864-70.

16. Fardon DF, Williams AL, Dohring EJ, Murtagh FR, Gabriel Rothman SL, Sze GK. Lumbar disc nomenclature: version 2.0: Recommendations of the combined task forces of the North American Spine Society, the American Society of Spine Radiology and the American Society of Neuroradiology. Spine J 2014;14:252545.

17. Vassiliev D. Spread of contrast during L4 and L5 nerve root infiltration under fluoroscopic guidance. Pain Physician 2007;10:461-6.

18. Ahn KH, Kim HS, Lee JH, Kim DH, Cho DI, Shin JC, et al. Effects of L4-5 transforaminal epidural steroid injection in L5 radiculopathy. J Korean Acad Rehabil Med 2005;29:281-5.

19. Furman MB, Mehta AR, Kim RE, Simon JI, Patel R, Lee TS, et al. Injectate volumes needed to reach specific landmarks in lumbar transforaminal epidural injections. PM R 2010;2:625-35.

20. Atluri S, Glaser SE, Shah RV, Sudarshan G. Needle position analysis in cases of paralysis from transforaminal epidurals: consider alternative approaches to traditional technique. Pain Physician 2013;16:321-34.

21. Lee HM, Kim NH, Kim HJ, Chung IH. Morphometric study of the lumbar spinal canal in the Korean population. Spine (Phila Pa 1976) 1995;20:1679-84.

22. Botwin K, Natalicchio J, Brown LA. Epidurography contrast patterns with fluoroscopic guided lumbar transforaminal epidural injections: a prospective evaluation. Pain Physician 2004;7:211-5.

23. Gajraj NM. Selective nerve root blocks for low back pain and radiculopathy. Reg Anesth Pain Med 2004; 29:243-56. 


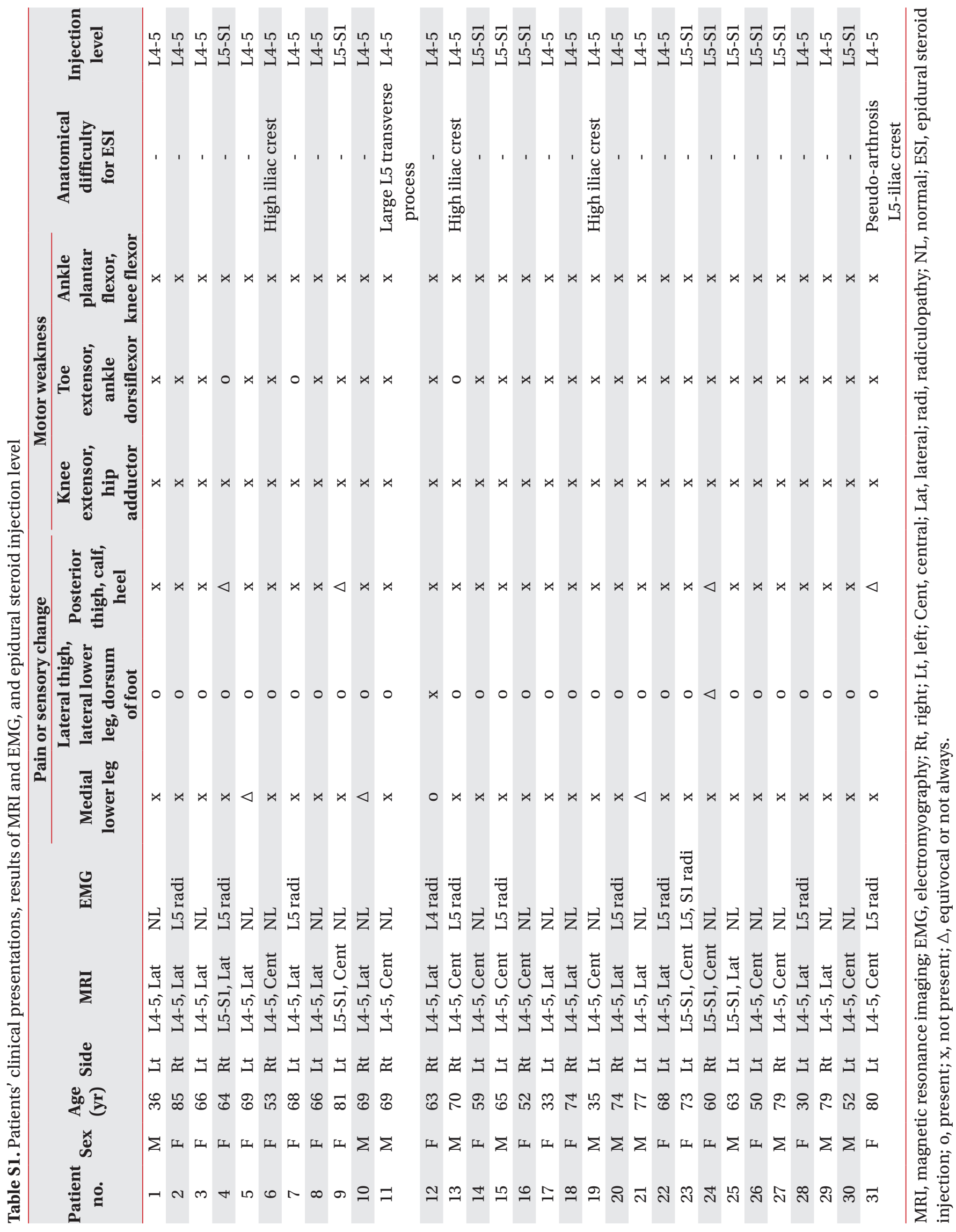

